\title{
Active Filter Implementation Using a Generalized Nonactive Power Theory
}

\author{
Yan $\mathrm{Xu}^{1}$ \\ yxu3@utk.edu
}

John N. Chiasson ${ }^{1}$

chiasson@utk.edu
Jeremy B. Campbell ${ }^{2}$

campbelljb@ornl.gov

Fang Z. Peng ${ }^{3}$

fzpeng@msu.edu

\author{
${ }^{3}$ Michigan State University \\ Electrical and Computer Engineering \\ East Lansing, MI 48824-1226
}

\begin{abstract}
This paper presents a generalized nonactive power theory, in which the instantaneous currents (active and nonactive) and instantaneous powers (active and nonactive) are defined. This theory is implemented in a parallel nonactive power compensation system. The theory is valid if the system is threephase or single-phase, sinusoidal or non-sinusoidal, periodic or non-periodic, balanced or unbalanced. Four cases, three-phase balanced RL load, three-phase unbalanced RL load, diode rectifier load, and single-phase RL load are tested in the experiments. A subharmonic load compensation and a nonperiodic load compensation are simulated in Matlab. The simulation and experimental results not only verify the validity of the theory, but also show that this theory can perform instantaneous nonactive power compensation with fast dynamic response.
\end{abstract}

Keywords - active filter, nonactive power, reactive power, STATCOM, harmonics, subharmonics, non-periodic

\section{INTRODUCTION}

Time-based instantaneous nonactive power theory was first formulated in the 1930's by Fryze [1], and the increasing rate of nonlinear loads requires a more comprehensive theory to describe, measure, and compensate these loads. However, most of the previous efforts have focused on periodic nonsinusoidal systems, rather than non-periodic systems [2]. The $p-q$ theory proposed in [3] is valid for three-phase three-wire systems with harmonics, and it has been extended to threephase four-wire systems [4-5]. In [6], Hilbert space techniques are adopted for the definition and compensation of reactive power. However, it introduces new harmonics to the active current which is not desired in nonactive power compensation. Non-integer multiple harmonics are defined as non-periodic currents in [7], and the compensation is discussed. Nonperiodic currents are also discussed in [8].

The diversity of the features of nonlinear currents makes it difficult to get one definition that fits all situations, and the compensation of such currents is quite difficult. The theory proposed in this paper is a generalized one which is independent of the number of phases in the system, applies whether the system is balanced or unbalanced, and to both periodic and non-periodic loads.
This generalized instantaneous nonactive power theory is applied to a STATCOM. The STATCOM provides the nonactive component of the load current which is calculated based on the generalized instantaneous nonactive power theory. A control scheme is presented to control the nonactive current and the DC link voltage as well. The experimental results of four different load cases show the validity of the nonactive power theory for active filtering.

\section{The Generalized NonACTIVE Power Theory}

The generalized instantaneous nonactive power theory [11] is an extension of the theory in [9]. For a voltage vector $\boldsymbol{v}(t)$ and a current vector $\boldsymbol{i}(t)$ (vectors for voltage and current will be denoted in bold),

$$
\begin{aligned}
& \boldsymbol{v}(t)=\left[v_{1}(t), v_{2}(t), \ldots, v_{m}(t)\right]^{T}, \\
& \boldsymbol{i}(t)=\left[i_{1}(t), i_{2}(t), \ldots, i_{m}(t)\right]^{T},
\end{aligned}
$$

where $m$ is the number of phases. The instantaneous power $p(t)$ is defined by

$$
p(t) \triangleq \boldsymbol{v}^{T}(t) \boldsymbol{i}(t)=\sum_{k=1}^{m} v_{k}(t) i_{k}(t) .
$$

The average power $P(t)$ is defined as the average value of $p(t)$ over the averaging interval $\left[t-T_{c}, t\right]$,

$$
P(t) \triangleq \frac{1}{T_{c}} \int_{t-T_{c}}^{t} p(\tau) d \tau .
$$

The averaging interval $T_{c}$ can be chosen arbitrarily from zero to infinity, and for different $T_{c}$, the resulting active current and nonactive current will have different characteristics. $T_{c}$ will be discussed in detail in Subsection $A$.

The instantaneous active current $i_{a}(t)$ and instantaneous nonactive current $\boldsymbol{i}_{n}(\boldsymbol{t})$ are defined by, respectively,

$$
\begin{aligned}
& \boldsymbol{i}_{a}(t) \triangleq \frac{P(t)}{V_{p}^{2}(t)} \boldsymbol{v}_{p}(t), \\
& \boldsymbol{i}_{n}(t) \triangleq i(t)-\boldsymbol{i}_{a}(t) .
\end{aligned}
$$


The voltage $\boldsymbol{v}_{\boldsymbol{p}}(t)$ is the reference voltage, which is chosen based on the characteristics of the system and the desired compensation results. It will be discussed in detail in Subsection $B . V_{p}(t)$ is the rms value of the reference voltage $v_{p}(t)$, i.e.,

$$
V_{p}(t) \triangleq \sqrt{\frac{1}{T_{c}} \int_{t-T_{c}}^{t} \boldsymbol{v}_{p}^{T}(\tau) \boldsymbol{v}_{p}(\tau) d \tau} .
$$

Based on the above definitions for $\boldsymbol{i}_{a}(t)$ and $\boldsymbol{i}_{\boldsymbol{n}}(t)$, the instantaneous active power $p_{a}(t)$ and instantaneous nonactive power $p_{n}(t)$ are defined by, respectively,

$$
\begin{aligned}
& p_{a}(t) \triangleq \boldsymbol{v}^{T}(t) \boldsymbol{i}_{\boldsymbol{a}}(t)=\sum_{k=1}^{m} v_{k}(t) i_{a k}(t) \\
& p_{n}(t) \triangleq \boldsymbol{v}^{T}(t) \boldsymbol{i}_{\boldsymbol{n}}(t)=\sum_{k=1}^{m} v_{k}(t) i_{n k}(t)
\end{aligned}
$$

The average active power $P_{a}(t)$ is defined as the average value of $p_{a}(t)$ over the averaging interval $\left[t-T_{c}, t\right]$,

$$
P_{a}(t) \triangleq \frac{1}{T_{c}} \int_{t-T_{c}}^{t} p_{a}(\tau) d \tau
$$

The average nonactive power $P_{n}(t)$ is defined as the average value of $p_{n}(t)$ over $\left[t-T_{c}, t\right]$,

$$
P_{n}(t) \triangleq \frac{1}{T_{c}} \int_{t-T_{c}}^{t} p_{n}(\tau) d \tau
$$

The definitions in this instantaneous nonactive power theory are consistent with the standard definitions for threephase fundamental sinusoidal systems, and valid in various cases, such as single-phase systems, non-sinusoidal systems, and non-periodic systems as well, by changing the averaging interval $T_{c}$ and the reference voltage $\boldsymbol{v}_{p}(t)$.

\section{A. Averaging Interval $T_{c}$}

The averaging time interval $T_{c}$ can be chosen arbitrarily from zero to infinity, and for different $T_{c}$, the resulting active current and nonactive current will have different characteristics. For each case, a specific value of $T_{c}$ can be chosen to fit the application or to achieve an optimal result. The choices of $T_{c}$ are:

1. $T_{c}=0$, the definitions of average powers are the same as the instantaneous powers, and the rms definitions have different forms, i.e.,

$$
\begin{aligned}
& V(t)=\boldsymbol{v}^{T}(t) \boldsymbol{v}(t), \\
& I(t)=\boldsymbol{i}^{T}(t) \boldsymbol{i}(t), \\
& V_{p}(t)=\boldsymbol{v}_{p}^{T}(t) \boldsymbol{v}_{p}(t) .
\end{aligned}
$$

The definitions of $\boldsymbol{i}_{\boldsymbol{a}}(t)$ and $\boldsymbol{i}_{\boldsymbol{n}}(t)$ have the same form as (5) and (6). If $\boldsymbol{v}_{p}(t)=\boldsymbol{v}(t)$, the instantaneous active power $p_{a}(t)$ is equal to the instantaneous power $p(t)$, and the instantaneous nonactive power $p_{n}(t)$ is identically zero. More specifically, in a single-phase system, the instantaneous active current $i_{a}(t)$ is always equal to the current $i(t)$, and the instantaneous nonactive current $i_{n}(t)$ is always zero, therefore, $T_{c}=0$ is not suitable for single-phase systems.

2. $T_{c}$ is a finite value. For most applications, $T_{c}$ will be chosen as a finite value. For a periodic system with fundamental period $T, T_{c}$ is chosen as $T_{c}=T / 2$. If $\boldsymbol{v}_{p}(\mathrm{t})$ is chosen as a periodic waveform with period $T$, then the average power $P(t)$ and the rms value $V_{p}(t)$ are both constant numbers, i.e., $P(t)=P$, and $V_{p}(t)=V_{p}$.

The instantaneous active current $\boldsymbol{i}_{\boldsymbol{a}}(t)$ is proportional to and has the same shape as the reference voltage. Therefore, by choosing different reference voltages, the instantaneous active current can have different waveforms.

3. $T_{c} \rightarrow \infty$. This is a theoretical analysis of a non-periodic system, in which a finite $T_{c}$ can not completely account for the entire nonactive component in the current.

The generalized nonactive power theory does not specify the characteristics of the voltage $\boldsymbol{v}(t)$ and current $i(t)$, i.e., they can theoretically be any waveforms. However in a power system, the voltage is usually sinusoidal with/without harmonic distortion, and the distortion of the voltage is usually lower than that of the currents (the total harmonic distortion (THD) of the voltage is usually less than $5 \%$.). Therefore in this paper, the voltage is assumed to be periodic for all cases. A non-periodic system is referred to as a system with periodic voltage and a non-periodic current.

In a non-periodic system, the instantaneous current varies with different averaging interval $T_{c}$, which is different from the periodic cases. The nonactive component in a non-periodic current will be completely subtracted by choosing $T_{c} \rightarrow \infty$. However, $T_{c} \rightarrow \infty$ is not practical in an actual power system application, and a finite $T_{c}$ will be used instead. In applications such as nonactive power compensation, $T_{c}$ is usually chosen to be 1-10 times that of the fundamental period based on the tradeoff between acceptable compensation results and reasonable capital costs.

\section{B. Reference Voltage $\boldsymbol{v}_{\boldsymbol{p}}(t)$}

If $P(t)$ and $V_{p}(t)$ are constant, which can be achieved with $T_{c}=T / 2$ for a periodic system, or with $T_{c} \rightarrow \infty$ for a nonperiodic system, the active current $\boldsymbol{i}_{\boldsymbol{a}}(t)$ is in phase with $\boldsymbol{v}_{p}(t)$, and the waveforms of $\boldsymbol{i}_{\boldsymbol{a}}(t)$ and $\boldsymbol{v}_{p}(t)$ have the same shape and they differ only by a scale factor. Theoretically, $v_{p}(t)$ can be arbitrarily chosen, but in practice, it is chosen based on the voltage $\boldsymbol{v}(t)$, the current $\boldsymbol{i}(t)$, and the desired active current $\boldsymbol{i}_{\boldsymbol{a}}(t)$. Choices for $v_{p}(t)$ include

1. $\boldsymbol{v}_{p}(t)=\boldsymbol{v}(t)$. If $\boldsymbol{v}(t)$ is a pure sinusoid; or the active current $\boldsymbol{i}_{\boldsymbol{a}}(t)$ is preferred to have the same waveform as $\boldsymbol{v}(t)$.

2. $v_{p}(t)=v_{f}(t)$, where $v_{f}(t)$ is the fundamental positive sequence component of $\boldsymbol{v}(t)$. In power systems, if $\boldsymbol{v}(t)$ is distorted or even unbalanced, and a purely sinusoidal $\boldsymbol{i}_{\boldsymbol{a}}(t)$ is desired, then $\boldsymbol{v}_{p}(t)$ is chosen as the fundamental positive sequence component of $\boldsymbol{v}(t)$. This ensures that $\boldsymbol{i}_{\boldsymbol{a}}(t)$ is balanced and does not contain any harmonics.

3. Other references to eliminate certain components in current $\boldsymbol{i}(t)$. For example, in a hybrid nonactive power compensation system with a STATCOM and a passive $L C$ 


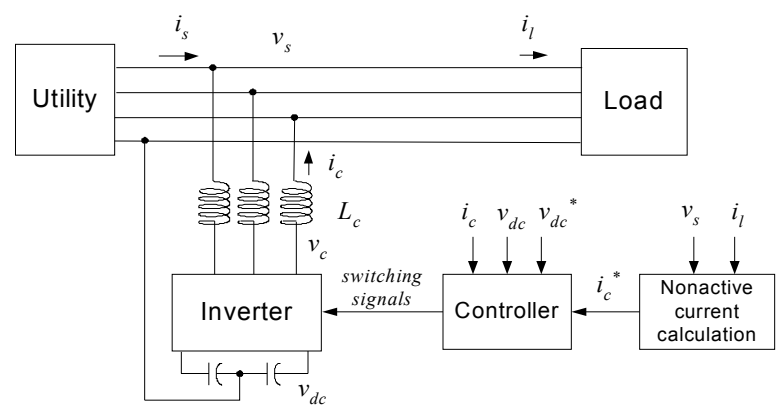

Fig. 1. System configuration of a parallel nonactive compensation system.

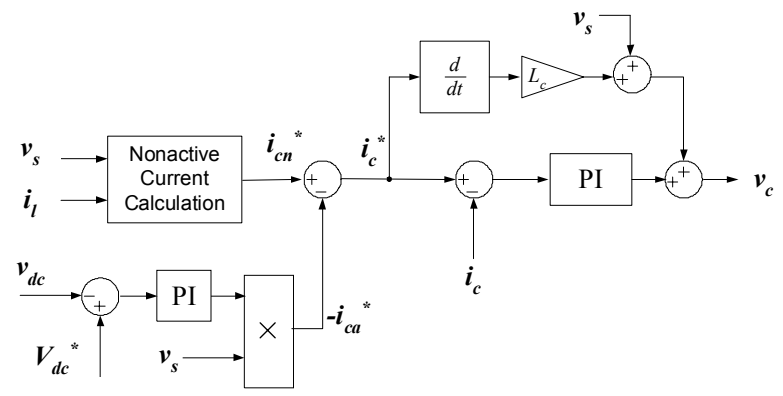

Fig. 2. Control diagram of the parallel nonactive compensator.

filter, the lower order harmonics in the current are compensated by the STATCOM, and the higher order harmonics will be filtered by the passive filter due to the limit of the switching frequency of the STATCOM. In this case, a reference voltage with the higher order harmonics is chosen so that the resulting nonactive current which will be compensated by the STATCOM does not contain these harmonics.

By choosing different reference voltages, $\boldsymbol{i}_{\boldsymbol{a}}(t)$ can have various desired waveforms, and the unwanted components in $i(t)$ can be eliminated. Furthermore, the elimination of each component is independent of each other.

\section{PARAlLel Nonactive Power COMPENSATION}

A parallel nonactive power compensator injects a certain amount of current, which is usually the nonactive component in the load current $\boldsymbol{i}_{n}(t)$, into the system so that the utility only provides the active component of the load current $\boldsymbol{i}_{\boldsymbol{a}}(t)$ [12]. In Fig. $1 \mathrm{a}, \boldsymbol{i}_{\boldsymbol{l}}$ is the load current, $\boldsymbol{i}_{\boldsymbol{s}}$ is the source current, and $\boldsymbol{i}_{\boldsymbol{c}}$ is the compensator current. Therefore, if the nonactive current is completely compensated by the compensator, then $\boldsymbol{i}_{\boldsymbol{s}}=\boldsymbol{i}_{a}(t)$, and $\boldsymbol{i}_{\boldsymbol{c}}=\boldsymbol{i}_{\boldsymbol{n}}(t)$. The generalized nonactive power theory is implemented in a parallel compensator, and the system configuration is shown in Fig. 1.

The system voltage $\boldsymbol{v}_{\boldsymbol{s}}$ and load current $\boldsymbol{i}_{\boldsymbol{l}}$ are measured and used to calculate the nonactive component of $\boldsymbol{i}_{l}$, which is the current the compensator needs to inject into the system $\left(\boldsymbol{i}_{c}{ }^{*}\right.$ in Fig. 1). In the theory presented in the previous section, the compensation system is assumed to be lossless; however in a real system, losses exist in the switches, capacitors, and inductors. If the compensator does not have any energy source, the energy stored in the DC link capacitor is drawn to compensate these losses, which will cause $v_{d c}$ to drop. The source voltage $\boldsymbol{v}_{\boldsymbol{s}}$ and load current $\boldsymbol{i}_{\boldsymbol{l}}$ are measured and the nonactive current $\boldsymbol{i}_{c}{ }^{*}$ is calculated. The active power required to replace the losses is drawn from the source by regulating the DC link voltage $v_{d c}$ to the reference $V_{d c}{ }^{*}$.

There are two components in the compensator current $\boldsymbol{i}_{c}$, one is the nonactive component $\boldsymbol{i}_{c n}$ to compensate the nonactive component of the load current, and the other is the active component $\boldsymbol{i}_{c a}$ to meet the compensator's losses by regulating the DC link voltage $v_{d c}$. A PI controller is used to regulate the DC link voltage $v_{d c}$ [10]. The active current $\boldsymbol{i}_{c a}$ required to meet the losses is in phase with $\boldsymbol{v}_{\boldsymbol{s}}$, and the amplitude of $\boldsymbol{i}_{c a}$ is controlled by the difference between the reference voltage $V_{d c}{ }^{*}$ and the actual DC link voltage $v_{d c} . \boldsymbol{i}_{c a}{ }^{*}$ is calculated by the amplitude modulation of $\boldsymbol{v}_{\boldsymbol{s}}$ as follows

$$
i_{c a}^{*}=-v_{s}\left[K_{P 1}\left(V_{d c}^{*}-v_{d c}\right)+K_{I 1} \int_{0}^{t}\left(V_{d c}^{*}-v_{d c}\right) d t\right] .
$$

According to the system configuration shown in Fig. 1, the relationship between the compensator's current $\boldsymbol{i}_{c}$ and the system voltage is

$$
L_{c} \frac{d \boldsymbol{i}_{c}}{d t}=\boldsymbol{v}_{c}-\boldsymbol{v}_{s} .
$$

A feedforward controller is designed as shown in (17).

$$
\boldsymbol{v}_{c}=\boldsymbol{v}_{s}+L_{c} \frac{d \boldsymbol{i}_{c}^{*}}{d t}+K_{P 2}\left(\boldsymbol{i}_{c}^{*}-\boldsymbol{i}_{c}\right)+K_{I 2} \int_{0}^{t}\left(\boldsymbol{i}_{c}^{*}-\boldsymbol{i}_{c}\right) d t
$$

Fig. 2 is the complete control diagram of the shunt compensation system. The inner loop is the compensator current control which controls the output voltage of the compensator according the required nonactive current $\boldsymbol{i}_{\boldsymbol{c}}{ }^{*}$. The outer loop controls the active current drawn by the compensator by regulating the DC link voltage $v_{d c}$, and combines the active current $\boldsymbol{i}_{c a}{ }^{*}$ together with the nonactive current $\boldsymbol{i}_{c n}{ }^{*}$.

\section{SimUlation AND EXPERIMENTAL RESUltS}

The nonactive power compensation system presented in Section III is simulated, and an experimental setup is also built, so that different cases can be studied in simulations or experiments. The fist four cases (Subsections $A-D$ ) are tested in the experimental setup, and the last two cases (Subsections $E$ and $F$ ) are simulated since they are difficult to be carried out in an experimental setup.

The system voltage of the utility is $208 \mathrm{~V}$ (rms value of line-to-line voltage). A Powerex POW-R-PAK ${ }^{\mathrm{TM}}$ configurable IGBT based three-phase inverter with a capacitor on the DC side is used to provide nonactive power. dSPACE, a real-time control platform, is used to implement the Simulink controller in hardware to perform the real-time control of the nonactive power compensator. The system voltage, load current, and compensator current are measured by potential transducers (PTs) and current transducers (CTs), and the measurements are fed to the dSPACE for calculation and control purposes. PWM 


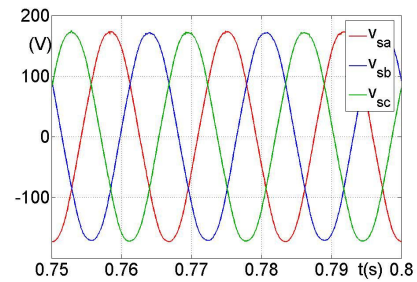

(a) System voltage

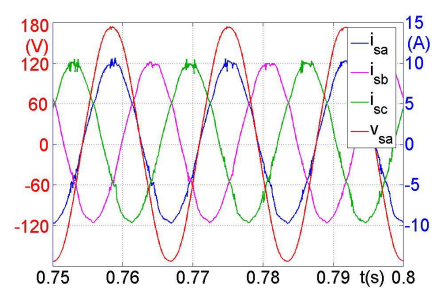

(c) Source current

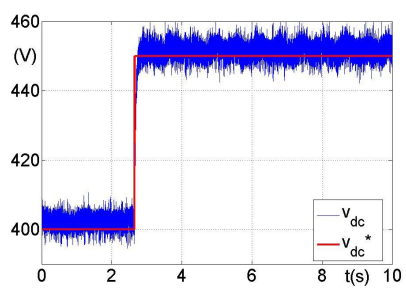

(e) DC link voltage change

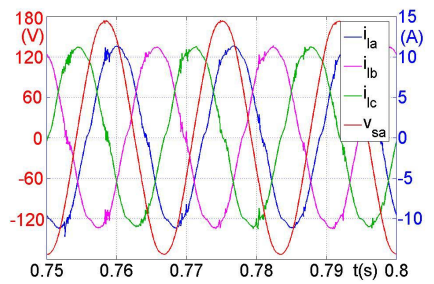

(b) Load current

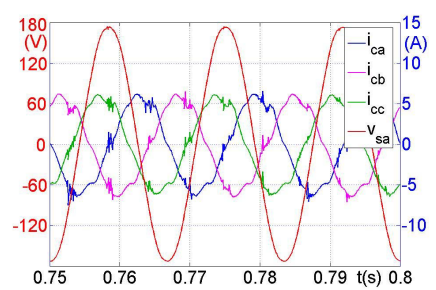

(d) Compensator current

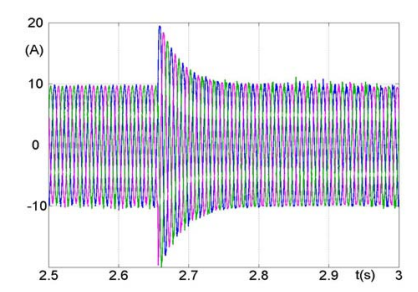

(f) Source current responding to the DC link voltage change
Fig. 3. Three-phase balanced RL load compensation.

control signals are generated by the dSPACE system to drive the switches of the inverter.

\section{A. Three-Phase Balanced RL Load}

In each phase, the resistor is $10.8 \Omega$, and the inductor is $20 \mathrm{mH}$. The three phase system voltage waveforms are shown in Fig. 3a. The load current waveforms are plotted in Fig. 3b, together with the phase $a$ system voltage to show the phase angle between the system voltage and the load current. Fig. 3c shows the source current after compensation, together with phase $a$ voltage. The amplitude of the source current is smaller than the load current, and the source current is in phase with the system voltage. The compensator current is illustrated in Fig. $3 \mathrm{~d}$. This current, which is about $90^{\circ}$ out of phase with the system voltage, contains mostly nonactive current. Before the compensation, the power factor of the load is 0.827 ; after compensation, the power factor of the source current is 0.998 . Therefore, the nonactive power compensator can improve the load power factor to nearly unity power factor.

Figs. $3 \mathrm{e}$ and $3 \mathrm{f}$ show the DC link voltage control. In Fig. $3 \mathrm{e}$, the red waveform is the reference DC voltage, which is $400 \mathrm{~V}$ at the beginning, and a step change to $450 \mathrm{~V}$ at $t=$ 2.66s. The blue waveform is the actual DC link voltage measured by the PT. To regulate the DC voltage from $400 \mathrm{~V}$ to $450 \mathrm{~V}$, the compensator draws a larger amount of active current than usual to charge the capacitor (as shown in Fig. 3f). This is performed by the DC link voltage PI controller. Because of the system constraints, for instance, the current and/or power ratings of the components in the compensator,

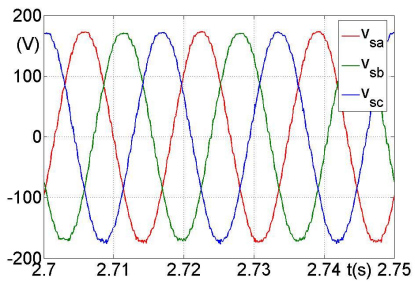

(a) System voltage $v_{s}(t)$

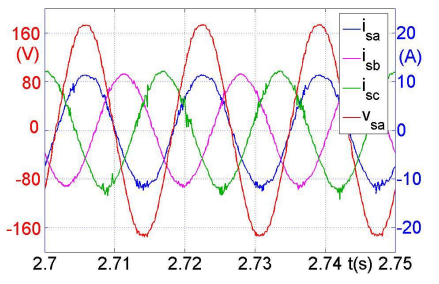

(c) Source current $i_{s}(t)$

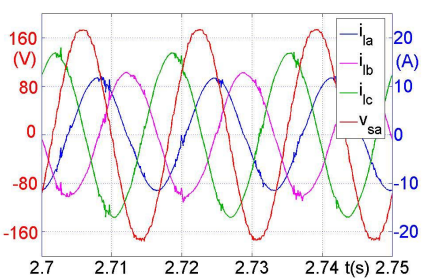

(b) Load current $\boldsymbol{i}_{l}(t)$

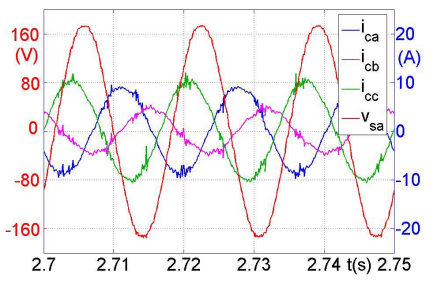

(d) Compensation current $\boldsymbol{i}_{c}(t)$
Fig. 4. Three-phase unbalanced RL load compensation.

only a limited current can be drawn, which causes the actual DC link voltage to reach the new reference value in about 0.1 s. Higher current rating can reduce this process and provide faster dynamic response.

\section{B. Three-Phase Unbalanced RL Load}

The inductors of the RL load are not equal in each phase, i.e., $30 \mathrm{mH}, 10 \mathrm{mH}$, and $10 \mathrm{mH}$, respectively. Therefore, the three phase load currents are not balanced any more, as shown in Fig. 4b. The three phase system voltages are balanced, as shown in Fig. 4a. The rms values of three phase load currents are $8.06 \mathrm{~A}, 9.00 \mathrm{~A}$, and $11.81 \mathrm{~A}$, respectively. Fig. 4c shows the source currents after compensation, which are more balanced than the load currents. The compensation current is shown in Fig. 4d. The rms values of the three phase source currents are $8.11 \mathrm{~A}, 7.95 \mathrm{~A}$, and $8.35 \mathrm{~A}$, respectively. The unbalance of a three-phase system is calculated as

$$
I_{\text {unbalance }}=\frac{\max \left\{\left|I_{a}-I_{b}\right|,\left|I_{b}-I_{c}\right|,\left|I_{c}-I_{a}\right|\right\}}{\left(I_{a}+I_{b}+I_{c}\right) / 3},
$$

The unbalance of the load current is $38.97 \%$, and the unbalance of the source current is improved to $4.92 \%$ after compensation.

\section{Three-Phase Diode Rectifier Load}

A three-phase diode rectifier is the load. A resistive load is used on the DC side of the three-phase diode rectifier. The highly distorted load current is shown in Fig 5b. Figs 5c and $5 \mathrm{~d}$ show the source current and compensator current when the DC link voltage is $500 \mathrm{~V}$. The source current is still distorted, which is mainly because of the large $d i / d t$ in the load current. A large $d i / d t$ requires large DC link voltage, i.e., a large inverter output voltage, so that the compensator can inject a large amount of current in a very short period. However, it is often not practical to have very high DC link voltage. Increasing the DC link voltage to $600 \mathrm{~V}$ and $700 \mathrm{~V}$, the resulting source currents are shown in Figs. 5e and 5f, respectively. The red sinusoidal waveforms in these figures are 


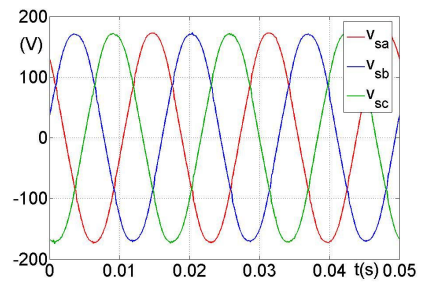

(a) System voltage $v_{s}(t)$

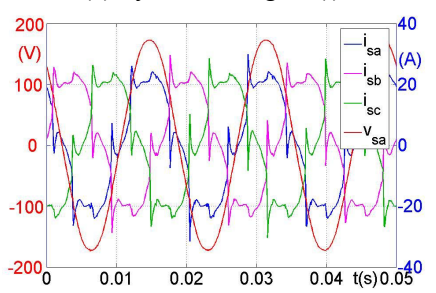

(c) Source current, $V_{d c}=500 \mathrm{~V}$

(e) Source current, $V_{d c}=600 \mathrm{~V}$

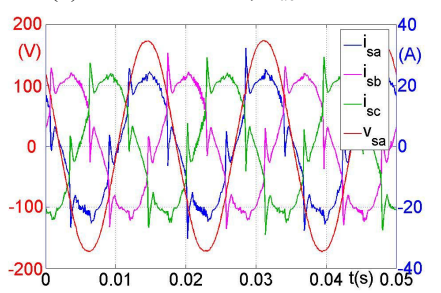

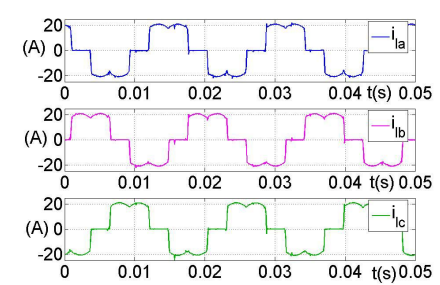

(b) Load current $i_{l}(t)$

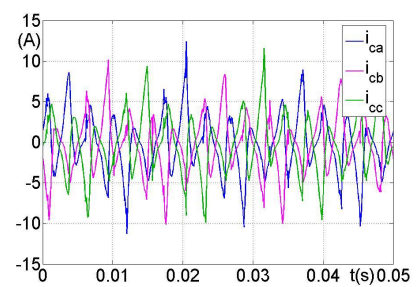

(d) Compensator current, $V_{d c}=500 \mathrm{~V}$

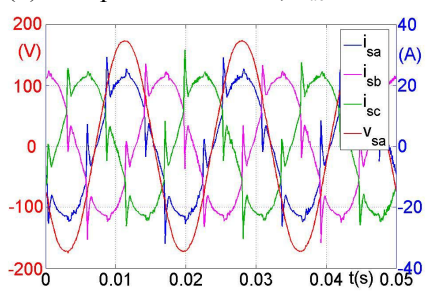

(f) Source current, $V_{d c}=700 \mathrm{~V}$
Fig. 5. Diode rectifier load compensation.

the phase $a$ voltage, to show that the currents are in phase with the voltage. By increasing the DC link voltage from $500 \mathrm{~V}$, to $600 \mathrm{~V}$, and then $700 \mathrm{~V}$, the waveforms of the source current are improving, i.e., closer to a sinusoid. The THD of the load current is $30.22 \%$. The THD of the source current is $25.32 \%$ when the DC link voltage is $500 \mathrm{~V}$, and it decreases to $21.24 \%$ and $18.84 \%$ when the DC link voltage is $600 \mathrm{~V}$ and $700 \mathrm{~V}$, respectively.

\section{Single-Phase Load}

An RL load is connected between phase $a$ and phase $b$ in the three-phase system while phase $c$ is left open (unloaded). The resistor is $29.2 \Omega$, and the inductor is $10 \mathrm{mH}$. The voltage and the load current are shown in Figs. 6a and 6b, respectively. The load current in phase $a$ and phase $b$ are equal magnitude and opposite direction, and the current in phase $c$ is zero. The rms values of the three phase load currents are 7.20 A, 7.22 A, and $0.43 \mathrm{~A}$, respectively. The source current and the compensator current are shown in Figs. $6 \mathrm{c}$ and $6 \mathrm{~d}$, respectively. The magnitudes of phase $a$ and phase $b$ source currents are reduced and there is a current in phase $c$. The rms values of the three phase source current are $4.44 \mathrm{~A}, 4.94 \mathrm{~A}$, and $3.97 \mathrm{~A}$, respectively. The values of phase $a$ and phase $b$ are reduced and the three phases are more balanced after compensation. The unbalance of the load current is $137.17 \%$, and the source current after compensation is $22.42 \%$.

\section{E. Three-Phase Load with Subharmonics}

The frequencies of subharmonics are not a multiple integer of the fundamental frequency. In this simulation, the

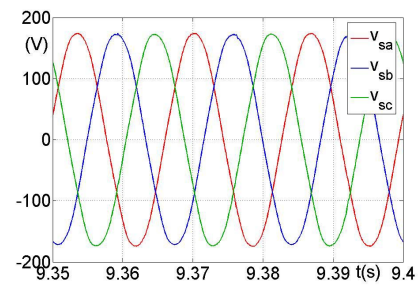

(a) System voltage $v_{s}(t)$

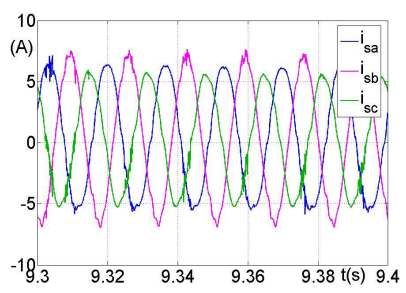

(c) Source current $\boldsymbol{i}_{\mathbf{s}}(t)$

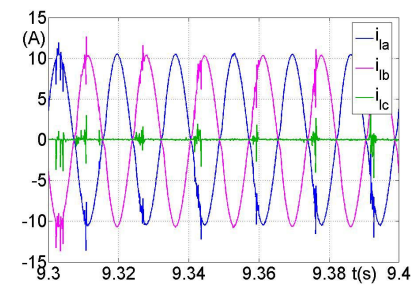

(b) Load current $\boldsymbol{i}_{l}(t)$

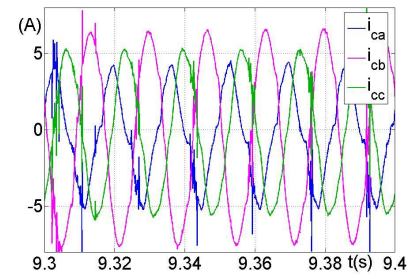

(d) Compensator current $\boldsymbol{i}_{c}(t)$
Fig. 6. Single-phase load compensation.

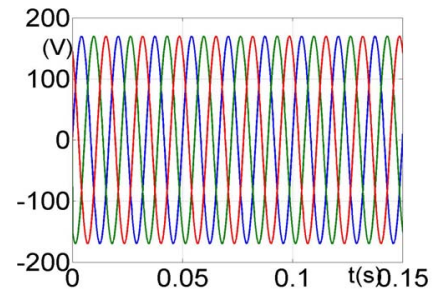

(a) Source voltage $v_{s}$

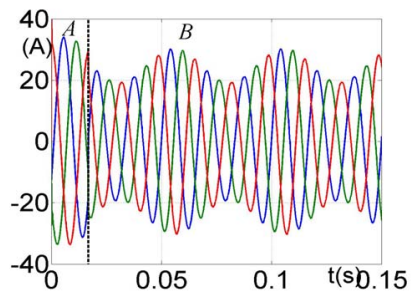

(c) Source current $\boldsymbol{i}_{\boldsymbol{s}}, T_{c}=T / 2$

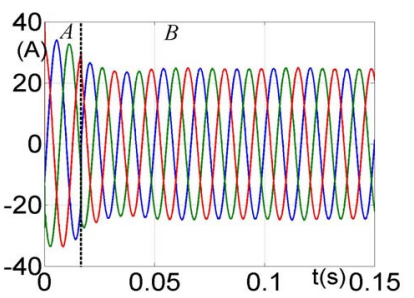

(e) Source current $\boldsymbol{i}_{\boldsymbol{s}}, T_{c}=3 T$

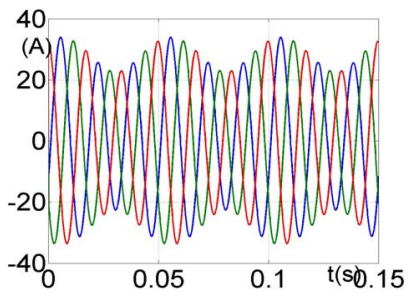

(b) Load current $\boldsymbol{i}_{l}$

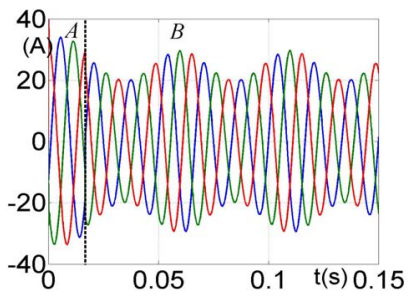

(d) Source current $\boldsymbol{i}_{\boldsymbol{s}}, T_{c}=T$
Fig. 7. Simulation of sub-harmonics load compensation.

voltage is a fundamental pure sine wave, and the load current has a sub-harmonic component with frequency $f_{s}=2 f / 3$, where $f$ is the fundamental frequency. The nonactive current is completely compensated if the averaging interval $T_{c}$ is a multiple integer of the common multiple of the fundamental component and the sub-harmonic component, which is $3 T$ in this case.

The simulation results are shown in Fig. 7. The system voltage $\boldsymbol{v}_{\boldsymbol{s}}(t)$ and load current $\boldsymbol{i}_{\boldsymbol{l}}(t)$ are shown in Figs. $7 \mathrm{a}$ and $7 \mathrm{~b}$ respectively. Figs. $7 \mathrm{c}-7 \mathrm{e}$ are the source current before and after 


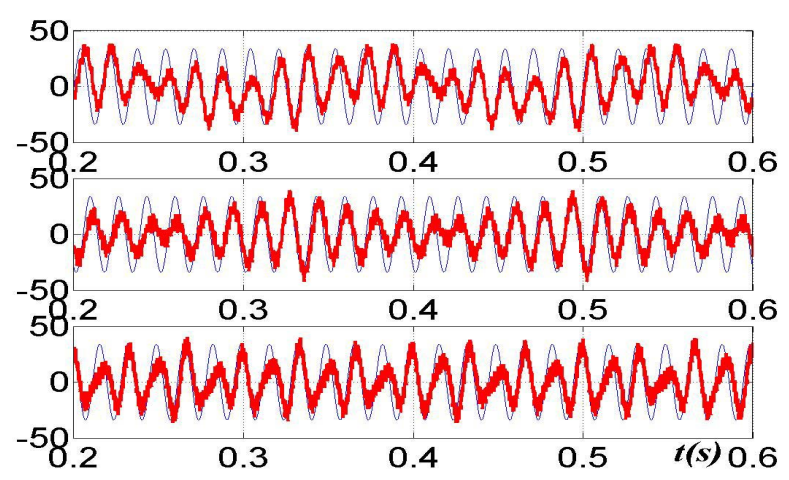

(a) Load current $i_{l}$ and system voltage $v_{s}$

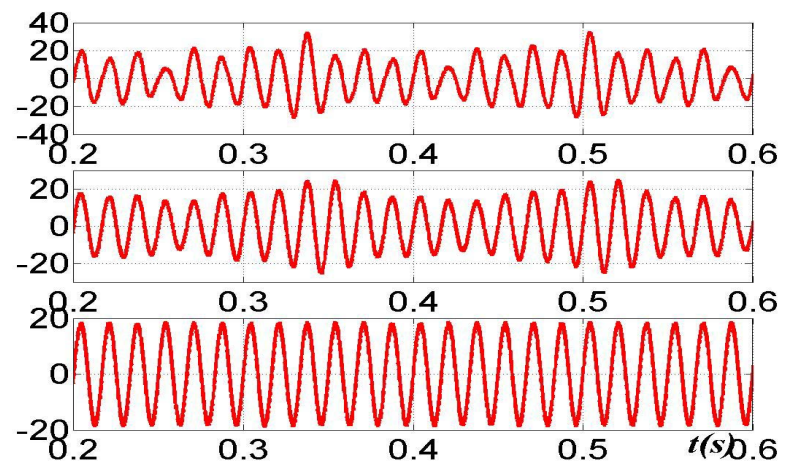

(b) Phase $a$ of source current $i_{s a}$ after compensation $T_{c}=T / 2,2 T$, and $10 T$

Fig. 8. Simulation of non-periodic load compensation.

compensation. The waveforms on the left side of the dashed lines (Region $A$ ) are the source currents before compensation, and the waveforms on the right side of the dashed lines (Region $B$ ) are the source current after compensation. Fig. 7c is the source current when $T_{c}=T / 2$. Fig. $7 \mathrm{~d}$ is the source current when $T_{c}=T$. The fundamental nonactive component is completely compensated, but the subharmonics component still exists. As illustrated, there are still subharmonics in the source current. The subharmonics are completely compensated if $T_{c}=3 T$ (for this example), as shown in Fig. 7e.

\section{F. Non-Periodic Load}

Theoretically, the period $T$ of a non-periodic load is infinite (a period much larger than the fundamental period of the utility) [13]. The nonactive components in a load cannot be completely compensated by choosing $T_{c}$ as $T / 2$ or $T$, or even several multiples of $T$. Fig. 8a shows three-phase non-periodic load current waveforms (red waveforms) and the system voltage waveforms (blue waveforms). Fig. $8 \mathrm{~b}$ shows the source currents after compensation, with $T_{c}=T / 2,2 T$, and 10T, respectively. With $T_{c}=T / 2$, there is still significant nonactive component in $i_{s}$ with variable peak values and a non-sinusoidal waveform. With $T_{c}=2 T$, the variation of the amplitude of $i_{s}$ is smaller, and with $T_{c}=10 T, i_{s}$ is close to a sine wave with less nonactive component. A longer $T_{c}$ "smoothes" the source current waveforms. Theoretically, $i_{s}$ could be a pure sine wave if $T_{c}$ goes to infinity, but in practice, such a $T_{c}$ cannot be implemented nor is it necessary. If $T_{c}$ is large enough, increasing $T_{c}$ further will not typically improve the compensation results significantly. For example, in the case shown in Fig. 8, the total harmonic distortion (THD) of $i_{s}$ is $6 \%$ with $T_{c}=2 T$, and with $T_{c}=10 T$, the THD is only slightly smaller (4\%). Typically, there is no need to increase $T_{c}$ to a larger value as the small decrease in THD is often not worth the larger capital costs (higher ratings of the compensator components and therefore higher capital expenses).

\section{DISCUSSION}

In nonactive power compensation, there are several factors which have significant influence on the choice of compensator type, the power rating of the compensator, the energy storage requirement of the compensator, and the compensation results. Some of these factors are related to the nonactive power theory itself, which include the averaging interval $T_{c}$, and the reference voltage $v_{p}$, while others are practical issues related to the implementation of the compensation system. In this paper, the coupling inductance $L_{c}$, the power rating of the compensator, and the capacitance of the DC link have been taken into consideration.

\section{A. Averaging Interval, $T_{c}$}

If there are only harmonics in the load current, $T_{c}$ does not change the compensation results as long as it is an integral multiple of $T / 2$, where $T$ is the fundamental period of the system. Here, the nonactive current is completely compensated, and a purely sinusoidal source current with a unity power factor is achieved.

However, in other cases, such as a three-phase load with subharmonics, or a non-periodic load, $T_{c}$ has significant influence on the compensation results, and the power and energy storage rating of the compensator's components. With longer $T_{c}$, a better source current will result, but at the cost of higher power rating for the switches and capacitance. There is a tradeoff between better compensation and higher system ratings (i.e., costs). On the other hand, a longer $T_{c}$ does not necessarily yield a significantly better source current waveform. For a specific system, there is an appropriate $T_{c}$ with which the compensation can be achieved. For example, in Subsection IV.E, $T_{c}$ depends on the frequency of the subharmonics.

\section{B. DC Link Voltage $v_{d c}$}

Generally, the compensator output voltage $v_{c}$ is the sum of the system voltage and the voltage drop on the coupling inductor $\left(L_{c}\right.$ in Fig. 1). The rms value of the required compensator output voltage $V_{c}$ at the worst situation, i.e., all the nonactive components reach the peak value at the same time is

$$
V_{c}=X_{c} I_{c}+V_{s} .
$$

The compensator current $I_{c}$ includes all the nonactive current components that are compensated, therefore, the reactance $X_{c}$ has different values for nonactive components of 
different frequencies. The higher order harmonics will have larger voltage drop on the inductance. The required DC link voltage $V_{d c}$ for a three-phase system is

$$
V_{d c}=2 \sqrt{2} V_{c} .
$$

The required DC link voltage $V_{d c}$ for a single-phase system is

$$
V_{d c}=\sqrt{2} V_{c} .
$$

\section{Coupling Inductance, $L_{c}$}

The coupling inductance $L_{c}$ between the power system and the compensator could be the inductance of a step-up transformer or a coupling reactor. It acts as the filter of the compensation current $i_{c}$, which has high ripple content due to the compensator's PWM control of the switches.

Generally a smaller $L_{c}$ is preferred, because a large $L_{c}$ requires a higher compensator output voltage, i.e., a higher DC link voltage. In practice, $L_{c}$ is chosen so that the fundamental voltage drop on this inductor is $3 \%-9 \%$ of the system voltage $V_{s}$. The inductance $L_{c}$ is

$$
L_{c}=(3 \%-9 \%) \frac{V_{s}}{\omega I_{c}} .
$$

\section{DC Link Capacitance Rating, $C$}

The DC link capacitance rating $C$ is proportional to the maximum energy storage variation of the capacitor. According to the discussion in Section II, the average power of the compensator $P_{c}(t)$ over $T_{c}$ is zero. Energy is neither generated nor consumed by the compensator, therefore, the energy stored in the capacitor is a constant at rated DC link voltage $V_{d c}$ given by

$$
E_{c}=\frac{1}{2} C V_{d c}{ }^{2} .
$$

However, the instantaneous power is not necessarily zero. The compensator generally has a capacitor for energy storage, and this capacitor operates in two modes, i.e., charge and discharge. Different capacitance values are required to fulfill different compensation tasks. The maximum energy variation in the capacitor is the integral of $v_{s}(t) i_{c}(t)$ between time $t_{\max }$ when the capacitor goes from discharge to charge, and $t_{\min }$ when the capacitor goes from charge to discharge, or vice versa.

$$
\Delta E_{c}=\int_{t_{\min }}^{t_{\max }} \boldsymbol{v}_{s}^{T}(t) \boldsymbol{i}_{c}(t) d t .
$$

The energy variation on the DC link capacitor causes the voltage variation, that is

$$
\Delta E_{c}=E_{c}\left(t_{\max }\right)-E_{c}\left(t_{\min }\right)=\frac{1}{2} C\left(V_{d c}^{2}\left(t_{\max }\right)-V_{d c}^{2}\left(t_{\min }\right)\right) .
$$

The required DC link capacitance $C$ is

$$
C=\frac{2 \Delta E_{c}}{V_{d c}^{2}\left(t_{\max }\right)-V_{d c}^{2}\left(t_{\min }\right)}=\frac{2 \int_{t_{\min }}^{t_{\max }} \boldsymbol{v}_{s}^{T}(t) \boldsymbol{i}_{c}(t) d t}{V_{d c}^{2}\left(t_{\max }\right)-V_{d c}^{2}\left(t_{\min }\right)} .
$$

For different applications, the energy variation $\Delta E_{c}$ is different, which determines the capacitance rating, for a given DC link voltage variation. In a three-phase RL load case, because the instantaneous nonactive power is zero at all times, the current flowing into or out of the DC link capacitor is zero. Therefore, a small capacitor can meet the requirement of this case. A large energy storage variation happens when the load is unbalanced, or there is a sudden load change, or a singlephase system, and a large capacitance is required.

\section{CONCLUSIONS}

A generalized nonactive power theory was presented in this paper for nonactive power compensation. The instantaneous active current, the instantaneous nonactive current, the instantaneous active power, and the instantaneous nonactive power were defined in a system which did not have any limitations such as the number of the phases, the voltage and the current were sinusoidal or non-sinusoidal, periodic or non-periodic. By changing the reference voltage and the averaging interval, this theory had the flexibility to define nonactive current and nonactive power in different cases. The flexibility was illustrated by applying the theory to different cases such as a sinusoidal system, a periodic system with harmonics, a periodic system with sub-harmonics, and a system with non-periodic currents.

This theory was implemented using a shunt compensator. The current that the compensator was required to provide was calculated based on the generalized nonactive power theory. A control scheme was developed to regulate the DC link voltage of the inverter, and to generate the switching signals for the inverter based on the required nonactive current. The compensation system for different cases was studied. The simulation and experimental results showed that the theory proposed in this paper was applicable to nonactive power compensation in three-phase systems, single-phase systems, load currents with harmonics, and non-periodic load currents. The DC link capacitance rating, the DC link voltage requirement, and the coupling inductance rating are determined in a three-phase RL load case, and these requirements for other cases are also discussed.

\section{ACKNOWLEDGEMENTS}

We would like to thank the National Science Foundation for supporting this work through contract NSF ECS-0093884.

\section{REFERENCES}

[1] S. Fryze, "Active, reactive, and apparent power in non-sinusoidal systems," Przeglad Elektrot, no. 7, 1931, pp. 193 - 203. (In Polish)

[2] L. M. Tolbert, T. G. Habetler, "Survey of active and non-active power definitions," IEEE International Power Electronics Congress, October 15-19, 2000, Acapulco, Mexico, pp. 73-79. 
[3] H. Akagi, Y. Kanazawa, A. Nabae, "Instantaneous reactive power compensators comprising switching devices without energy storage components," IEEE Trans. Ind. Appl., vol. 20, May/June 1984, pp. 625630.

[4] F. Z. Peng, J. S. Lai, "Generalized instantaneous reactive power theory for three-phase power systems," IEEE Transactions on Instrumentation and Measurement, Vol. 45, Feb. 1996, pp. 293 - 297.

[5] H. Akagi, "Active filters and energy storage systems operated under nonperiodic conditions," IEEE Power Engineering Society Summer Meeting, Seattle, Washington, July 15-20, 2000, pp. 965-970.

[6] H. Lev-Ari, A. M. Stankovic, "Hilbert space techniques for modelling and compensation of reactive power in energy processing systems," IEEE Transactions on Circuits and Systems, vol. 50, April 2003, pp. 540 $-556$.

[7] E. H. Watanabe, M. Aredes, "Compensation of non-periodic currents using the instantaneous power theory," IEEE Power Engineering Society Summer Meeting, Seattle, Washington, July 15-20, 2000, pp. 994-999.

[8] L. S. Czarnecki, "Non-periodic currents: their properties, identification and compensation fundamentals," IEEE Power Engineering Society Summer Meeting, Seattle, Washington, July 15-20, 2000, pp. 971-976.

[9] F. Z. Peng, L. M. Tolbert, "Compensation of non-active current in power systems - definitions from compensation standpoint," IEEE PowerEngineering Society Summer Meeting, July 15-20, 2000, Seattle, Washington, pp. $983-987$.

[10] M. D. Manjrekar, P. Steimer, T. A. Lipo, "Hybrid multilevel power conversion system: a competitive solution for high power applications," IEEE Transactions on Industry Applications, Vol. 36, May-June 2000, pp. $834-841$.

[11] Y. Xu, L. M. Tolbert, F. Z. Peng, J. N. Chiasson, J. Chen, "Compensation-based non-active power definition," IEEE Power Electronics Letters, vol. 1, no. 2, June 2003, pp. 45-50.

[12] Y. Xu, L. M. Tolbert, J. N. Chiasson, F. Z. Peng, "Dynamic response of active filter using a generalized nonactive power theory," IEEE Industry Applications Society Annual Meeting, October 2-6, 2005, Hong Kong, China, pp. 1225-1231.

[13] L. M. Tolbert, Y. Xu, J. Chen, F. Z. Peng, J. N. Chiasson, "Compensation of irregular currents with active filters," IEEE Power Engineering Society General Meeting, July 13-18, 2003, Toronto, Canada, pp. 1278-1283. 